\title{
A Symbolic-Numerical Envelope Algorithm Using Quadratic MOS Patches
}

\author{
Bohumír Bastl \\ University of West Bohemia, \\ Department of Mathematics \\ Univerzitní 8 \\ 30100 PIzeň, Czech Republic \\ bastl@kma.zcu.cz
}

\author{
Jiří Kosinka \\ University of Oslo, Centre of \\ Mathematics for Applications \\ P.O. Box 1053 Blindern \\ NO-0316 Oslo, Norway \\ jiri.kosinka@cma.uio.no
}

\author{
Miroslav Lávička \\ University of West Bohemia, \\ Department of Mathematics \\ Univerzitní 8 \\ 30100 Plzeň, Czech Republic \\ lavicka@kma.zcu.cz
}

\begin{abstract}
In this paper, we describe an algorithm for generating an exact rational envelope of a two-parameter family of spheres given by a quadratic patch in $\mathbb{R}^{3,1}$, which is considered as a medial surface transform (MST) of a spatial domain. Recently, it has been proved that quadratic triangular Bézier patches in $\mathbb{R}^{3,1}$ belong to the class of MOS surfaces (i.e., surfaces providing rational envelopes of the associated twoparameter family of spheres). We give a detailed description of the symbolic and numerical steps of the envelope algorithm and study the error involved in the numerical part. The presented method is then demonstrated on several examples. Moreover, since quadratic MOS patches are capable of producing $C^{1}$ approximations of MSTs, this algorithm offers a good basis for consequent methods, e.g. computing rational approximations of envelopes associated to general (free-form) MSTs and inner offsets trimming.
\end{abstract}

\section{Keywords}

MOS surfaces, quadratic patches, envelope formula, Bézier clipping, inner offsets, trimming

\section{INTRODUCTION}

Since the introduction of the medial axis transform (MAT) of a planar domain in [4], considerable interest has been devoted to this notion in various applications dealing with geometry of shapes such as Computational Geometry, Computer Vision, Pattern and Image Analysis, Computer Aided Design, etc. (see $[8,11,35,24,6,5,1]$ ). There is a one-toone correspondence between the MAT and the object boundary, i.e., for a given geometric object there is a unique MAT and conversely, the object can be directly reconstructed from its MAT. The main advantages of this description follow from the dimensional reduction while topological properties are maintained.

\footnotetext{
${ }^{*}$ Corresponding author.
}

In the spatial case, the medial surface transform (MST) of a domain is the set of surface patches (or curve segments) in four-dimensional space such that each point of these surfaces/curves represents the center and the radius of a maximal sphere inscribed into the domain. The MST covers the structure of the domain and thus can be used in all sorts of geometric modelers (constructive solid geometry (CSG) and boundary representation (B-rep)) as a useful tool.

Rational descriptions of geometric objects (NURBS) have become a universal standard in technical applications such as Computer Aided Design and Manufacturing. However, starting from a rational medial axis (surface) transform, the rationality of corresponding envelopes of the one- (two-) parameter family of circles (spheres) is generally not guaranteed. Moreover, the direct computation of the envelope leads to an implicit representation and thus it is challenging to decide on their rationality. Study of the rationality of envelopes of one-parameter families of circles in the plane led to the class of Minkowski Pythagorean hodograph (MPH) curves introduced in [26] as an extension of (classical) Pythagorean hodograph curves $[13,14]$. As observed in $[9,26]$, if a segment of the medial axis transform is an MPH curve, then the associated branches of the domain boundaries are segments of rational curves. Moreover, all offsets of the domain boundaries have also this property. A thorough analysis of MPH curves has followed - see [7, 10, 16, 17, 19].

The situation in three-dimensional space is more complicated and thus the study started by the analysis of envelopes of one-parameter families of spheres. This leads to pipe and canal surfaces, and especially to Dupin cyclides. The cyclographic model of Laguerre geometry turned out to be a useful tool for this analysis (see [34, 31, 20, 21]). Recently, the so called MOS surfaces as a spatial analogy of MPH curves in four-dimensional Minkowski space $\mathbb{R}^{3,1}$ were introduced in [18]. MOS surfaces extend the so-called PN (Pythagorean normal) surfaces defined in [33] and later studied e.g. in [25, $31,22,23]$. The distinguishing property of MOS surfaces is that if considered as an MST of a spatial domain, the associated envelope and its offsets admit exact rational parameterization. Later, it was proved in [29] that quadratic triangular Bézier surfaces in $\mathbb{R}^{3,1}$ possess the MOS property and a related study followed in [30].

In this paper, we describe an algorithm for computing an exact rational envelope of a two-parameter family of spheres 
given by a quadratic patch in $\mathbb{R}^{3,1}$ which is considered as the MST of a spatial domain. Following the results of [29], [2] and [3], we continue to study quadratic Bézier triangles from the point of view of their applications to computing exact rational parameterizations or approximations of offsets of spatial domains. The envelope algorithm consists of three main steps: subdividing the parameter domain, covering the isotropic Gauss image of a quadratic patch and parameterizing the envelope and its trimming. Since a numerical subdivision is needed for some classes of patches, we also give an analysis of the error involved. We are motivated by the fact that approximation by quadratic Bézier patches provides a good compromise between curvature reproduction and computational cost and offers an extra feature the MOS property. Moreover, the algorithm can be used as a foundation for future research on approximating free form MSTs, trimming of inner offsets of envelopes and other applications.

The remainder of this paper is organized as follows. Section 2 recalls some basic facts concerning medial surface transforms and MOS surfaces. Section 3 is devoted to isotropic normal vectors and isotropic Gauss image of quadratic Bézier triangles. In Section 4 we study the steps of the algorithm for envelope computation for quadratic MSTs. In Section 5 we give a summary of this aglorithm and mention some of its applications. Finally, the algorithm is demonstrated on several examples in Section 6 .

\section{PRELIMINARIES}

In this section we summarize some fundamental notions and basic properties of quadratic MOS patches in $\mathbb{R}^{3,1}$. The reader is kindly referred to $[3,18]$ for more details.

\subsection{Medial surfaces transforms}

A four-dimensional real affine space along with the indefinite bilinear form

$$
\langle\mathbf{u}, \mathbf{v}\rangle=\mathbf{u}^{\top} \mathbf{J} \mathbf{v}=u_{1} v_{1}+u_{2} v_{2}+u_{3} v_{3}-u_{4} v_{4},
$$

given by $\mathbf{J}=\operatorname{diag}(1,1,1,-1)$, is called Minkowski space and denoted $\mathbb{R}^{3,1}$. A linear subspace of $\mathbb{R}^{3,1}$ is called space-, time- or light-like if the restriction of the quadratic form defined by $\mathbf{J}$ is positive definite, indefinite nondegenerate or degenerate, respectively.

Points in the projective closure of $\mathbb{R}^{3,1}$ will be described using standard homogeneous coordinates

$$
x_{0}: x_{1}: x_{2}: x_{3}: x_{4}=1: x: y: z: r .
$$

The equation $x_{0}=0$ describes the ideal hyperplane as a set of all asymptotic directions, i.e., of points at infinity. The subset of the ideal hyperplane which is invariant with respect to transformations maintaining Minkowski inner product (Lorentz transforms) is called the absolute quadric $\Sigma$ and characterized by

$$
\Sigma:\langle\mathbf{x}, \mathbf{x}\rangle=x_{1}^{2}+x_{2}^{2}+x_{3}^{2}-x_{4}^{2}=x_{0}=0 .
$$

Consider a spatial domain $\Omega$ and the family of all inscribed spheres partially ordered with respect to inclusion of the associated balls. An inscribed sphere is called maximal if it is not contained in any other inscribed sphere. Then the medial surface (MS) of $\Omega$ is the locus of all centers $(x, y, z)^{\top}$ of maximal inscribed spheres and the medial surface transform (MST) of $\Omega$ is obtained by appending the corresponding sphere radius $r$ to the medial surface, i.e., it consists of points $(x, y, z, r)^{\top} \in \mathbb{R}^{3,1}$.

\subsection{MOS surfaces in Minkowski space}

Now, we consider the 2-parameter family of spheres

$$
(X-x(u, v))^{2}+(Y-y(u, v))^{2}+(Z-z(u, v))^{2}=r^{2}(u, v),
$$

which is regarded as a $C^{1}$ patch of a medial surface transform $A$ parameterized by $\mathbf{a}(u, v)=(x, y, z, r)^{\top}(u, v)$ over a parameter domain $\mathcal{D} \subseteq \mathbb{R}^{2}$. We denote by $\hat{\mathbf{a}}(u, v)=$ $(x, y, z)^{\top}(u, v)$ the corresponding medial surface.

The coordinates of a point $\mathbf{b}=(X, Y, Z)^{\top}$ on the envelope surface $B$ can be found by intersecting the sphere (4) with the two planes

$$
\begin{aligned}
& (X-x) \cdot x_{u}+(Y-y) \cdot y_{u}+(Z-z) \cdot z_{u}+r \cdot r_{u}=0 \\
& (X-x) \cdot x_{v}+(Y-y) \cdot y_{v}+(Z-z) \cdot z_{v}+r \cdot r_{v}=0 .
\end{aligned}
$$

It yields (cf. [18]) the closed-form envelope formula

$$
\mathbf{b}^{ \pm}(u, v)=\hat{\mathbf{a}}(u, v)+\frac{r}{\hat{C}}\left(\mathbf{w} \pm \sqrt{C} \cdot\left(\hat{\mathbf{a}}_{u} \times \hat{\mathbf{a}}_{v}\right)\right),
$$

where

$$
\hat{C}=\hat{E} \hat{G}-\hat{F}^{2}, \quad C=E G-F^{2} .
$$

The components $E, F, G$ of the first fundamental form of $\mathbf{a}(u, v)$ are computed using the Minkowski inner product in $\mathbb{R}^{3,1}$, whereas the components $\hat{E}, \hat{F}, \hat{G}$ of the first fundamental form of $\hat{\mathbf{a}}(u, v)$ are determined using the standard Euclidean inner product in $\mathbb{R}^{3}$. The vector $\mathbf{w}=\mathbf{w}(u, v)$ in (6) consists of polynomials of degree four in $x_{u}, y_{u}, z_{u}, r_{u}$ and $x_{v}, y_{v}, z_{v}, r_{v}$, see [18] for details.

Since the formula (6) contains the expression $\sqrt{C}$, we restrict the parameter domain to obtain a real envelope $B$.

Definition 1. The subset given by $C=E G-F^{2}>0$ in the parameter domain $\mathcal{D}$ is called the space-like domain of the surface patch. Its boundary is the curve $C(u, v)=0$, which corresponds to points of $\mathbf{a}(u, v)$ with light-like tangent planes.

Remark 1. The envelope formula is not well defined when

$$
\hat{E} \hat{G}-\hat{F}^{2}=0,
$$

i.e., at the singular points of $\hat{\mathbf{a}}(u, v)$. However, it can be proved that $\hat{\mathbf{a}}(u, v)$ has no singular points over the spacelike domain - see [3] for more details.

Definition 2. Let $A$ be an MST in $\mathbb{R}^{3,1}$ given by a polynomial or rational parameterization $\mathbf{a}(u, v)$. This parameterization is called an MOS parameterization if there exists a bivariate polynomial or rational function $\sigma(u, v)$, such that it holds

$$
E G-F^{2}=\sigma(u, v)^{2} .
$$

Then, $A$ is called an MOS surface if it possesses an MOS parameterization. 
MOS surfaces are a spatial analogy of Minkowski Pythagorean hodograph curves, see [9, 26], as both pieces of the boundary of the domain corresponding to an MOS surface patch (and their offsets) possess an exact rational parametric representation. This fact is fundamental for the symbolic part of our algorithm.

\subsection{Quadratic Bézier patches}

A quadratic patch in $\mathbb{R}^{3,1}$ is defined by a Bernstein-Bézier representation

$$
\mathbf{a}(u, v)=\sum_{\substack{i, j, k \geq 0 \\ i+j+k=2}} \mathbf{p}_{i j k} \frac{2}{i ! j ! k !} u^{i} v^{j}(1-u-v)^{k},
$$

where the parameters $u, v$ span a domain $\mathcal{D}$, see [12]. We will mostly work with the standard triangular domain $\triangle \subset \mathbb{R}^{2}$ obtained for $u \in[0,1]$ and $v \in[0,1-u]$. Sometimes it is more convenient to use the power basis representation

$$
\mathbf{a}(u, v)=\frac{1}{2} \mathbf{a}_{20} u^{2}+\mathbf{a}_{11} u v+\frac{1}{2} \mathbf{a}_{02} v^{2}+\mathbf{a}_{10} u+\mathbf{a}_{01} v+\mathbf{a}_{00} .
$$

Our aim is to find a rational reparameterization of $\mathbf{a}(u, v)$ leading to an MOS parameterization and thus we consider only such quadratic parameterizations which do not fulfill the MOS condition. Therefore, all surfaces lying in lightlike hyperplanes (cf. [18]) are excluded from our further considerations.

\section{ISOTROPIC GAUSS IMAGE}

At regular points, the normal vectors (with respect to Minkowski inner product) of the quadratic patch (11) satisfy the linear equations

$$
\left\langle\mathbf{n}, \mathbf{a}_{u}(u, v)\right\rangle=0, \quad\left\langle\mathbf{n}, \mathbf{a}_{v}(u, v)\right\rangle=0,
$$

where

$$
\begin{aligned}
& \mathbf{a}_{u}=\mathbf{a}_{20} u+\mathbf{a}_{11} v+\mathbf{a}_{10}, \\
& \mathbf{a}_{v}=\mathbf{a}_{11} u+\mathbf{a}_{02} v+\mathbf{a}_{01} .
\end{aligned}
$$

Among them, the isotropic normal vectors are characterized by

$$
\langle\mathbf{n}, \mathbf{n}\rangle=0 \text {. }
$$

As shown in [3], isotropic normal vectors of $\mathbf{a}(u, v)$ can be computed from the envelope formula (6) as

$$
\mathbf{n}^{ \pm}=\left(-\mathbf{w} \mp \sqrt{C}\left(\hat{\mathbf{a}}_{u} \times \hat{\mathbf{a}}_{v}\right), \hat{C}\right)^{\top} .
$$

Lemma 1. If $C=E G-F^{2}$ is positive, zero, or negative, then we obtain two, one, or no real isotropic normal vectors, respectively. In addition, $\mathbf{n}^{ \pm}(u, v)$ is rational if and only if the MOS condition (9) is fulfilled.

Following the results of [29], any quadratic patch in $\mathbb{R}^{3,1}$ can be reparameterized so that it fulfills the MOS condition. The reparameterization $(u, v)=\phi(s, t)$ is computed as a solution to the system of linear equations (12) in $u, v$. We set for $\mathbf{n}$ a suitable rational parameterization $\mathbf{n}(s, t)$ of the absolute quadric (14) and obtain

$$
\phi: \quad u=\frac{d_{1}(s, t)}{d(s, t)}, \quad v=\frac{d_{2}(s, t)}{d(s, t)},
$$

where $d, d_{1}$ and $d_{2}$ are the determinants involved in Cramer's rule applied to the linear system (12).

Next, given an isotropic vector $\mathbf{n}$ we consider all points on the quadratic surface $\mathbf{a}(u, v)$ (and corresponding parameters $(u, v)$ in the space-like domain) such that $\mathbf{n}$ is the isotropic normal vector at these points. Especially, we are interested in points not possessing the uniqueness property with respect to isotropic normals, i.e., at least one of the two isotropic normal vectors is an isotropic normal vector for another point on the surface.

Table 1: Canonical representants of affine classes, the type of the curve $\mathcal{E}$ and the maximum number $N$ of exceptional normals of each class.

\begin{tabular}{|c|c|c|c|}
\hline Type & $\mathbf{a}(u, v)$ & $\mathcal{E}$ & $N$ \\
\hline \hline (i) & $\left(u^{2}, v^{2}, u, v\right)^{\top}$ & 2 straight lines & $2+2=4$ \\
\hline (ii) & $\left(u^{2}, u v, u, v\right)^{\top}$ & 1 straight line & 2 \\
\hline (iii) & $\left(u^{2}-v^{2}, u v, u, v\right)^{\top}$ & 1 straight line & 2 \\
\hline (iv) & $\left(u^{2}, v^{2}, u v, u\right)^{\top}$ & line and conic & $2+4=6$ \\
\hline (v) & $\left(u^{2}+v, v^{2}, u v, u\right)^{\top}$ & irreducible cubic & 6 \\
\hline
\end{tabular}

The necessary condition for the unique solution of the system (12) is $d \neq 0$, where $d$ is the determinant of the system. Hence, we consider the equations

$$
\begin{gathered}
d=0, \\
\langle\mathbf{n}, \mathbf{n}\rangle=0,
\end{gathered}
$$

which describe a curve of degree four on the oval quadric $\Sigma$. Its real part will be called the singular curve and denoted $\mathcal{S}_{\Sigma}$. From the theory of linear systems we obtain:

Lemma 2. Each point of $\mathcal{S}_{\Sigma}$ corresponds to such an isotropic normal vector for which the system (12) has no or infinitely many solutions. In the latter case, the associated parameters $(u, v) \in \mathcal{D}$ form a straight line.

Definition 3. The straight lines involved in Lemma 2 are called exceptional lines. They form a set denoted by $\mathcal{K}$. The associated isotropic normal vectors are exceptional normals.

Remark 2. Non-developable quadratic surfaces lying in a space-like hyperplane were thoroughly studied in [2]. In this case, the exceptional lines correspond to preimages of parabolic lines and can be found by solving a certain cubic equation $P(u, v)=0$. Thus their maximum number is 3 . An analogous approach yields the same maximum number of exceptional lines for quadratic patches lying in time-like hyperplanes. On the other hand, surfaces lying in a light-like hyperplane were excluded from our considerations.

In order to find the exceptional lines for non-hyperplanar $\mathbf{a}(u, v)$, we consider the system

$$
d=0, d_{1}=0, d_{2}=0
$$


for $\mathbf{n}$, where $d, d_{1}$ and $d_{2}$ are as in (16). This system yields all normal vectors without the uniqueness property, not only the isotropic ones - to finish the computation we have to add the equation of the absolute quadric (14).

The system (18) defines a space curve $\mathcal{E}$ in the ideal hyperplane whose type is invariant with respect to affine mappings in $\mathbb{R}^{4}$. Hence, we recall the affine classification of quadratic patches, cf. [32]. The canonical representants of the 5 classes of non-hyperplanar quadratically parameterized surfaces and the corresponding type of $\mathcal{E}$ are presented in Table 1 (for the sake of brevity we omit the details of computing $\mathcal{E})$.

Lemma 3. There exist at most 6 exceptional lines for a non-hyperplanar quadratic patch $\mathbf{a}(u, v)$.

Proof. The spatial curve $\mathcal{E}$ is determined for each canonical representant (i)-(v), cf. Table 1 . Next, column 4 lists the maximum number of intersections of $\mathcal{E}$ and the absolute quadric and thus the maximum number of exceptional lines.

Remark 3. We emphasize that for the class (v), on the contrary to the remaining classes (and also hyperplanar patches), the exceptional lines cannot be, in general, computed using radicals since the determining equation $P(u, v)=0$ is of degree 6 . This fact accounts for the numerical part of our algorithm.

In what follows, the isotropic normals $\mathbf{n}$ will be identified with points of the quadric (14) considered as the unit sphere in $\mathbb{R}^{3}$.

Definition 4. We restrict the quadratic surface patch $\mathbf{a}(u, v)$ to the closure of its space-like domain. For each point we have two isotropic normal vectors $\mathbf{n}^{ \pm}$, which correspond to two points on $\Sigma$. The set of these points forms two components $\mathcal{G}^{ \pm}$, which will be called the isotropic Gauss image of $\mathbf{a}(u, v)$.

Several examples of the isotropic Gauss image of quadratic patches are shown in Fig. 3. As a consequence of the definition of exceptional normals we have:

LEMma 4. Exceptional normals of $\mathbf{a}(u, v)$ correspond to singularities of the associated isotropic Gauss image.

The following Lemma describes the connection between isotropic normals and exceptional lines and plays an important role in our envelope algorithm.

Lemma 5. For non-hyperplanar $\mathbf{a}(u, v)$, two different exceptional normals correspond to distinct exceptional lines. For $\mathbf{a}(u, v)$ lying in a space- or time-like hyperplane, each exceptional line corresponds to two distinct exceptional normals.
Proof. Computing the exceptional normals (cf. Table 1) reveals that two distinct exceptional normals cannot correspond to the same exceptional line of non-hyperplanar $\mathbf{a}(u, v)$. On the other hand, observe from (15) that the necessary condition for an isotropic normal to be constant along an exceptional line is $E G-F^{2}=\sigma(t)^{2}$, hence

$$
\mathbf{n}^{ \pm}=\left(-\mathbf{w} \mp \sigma\left(\hat{\mathbf{a}}_{u} \times \hat{\mathbf{a}}_{v}\right), \hat{C}\right)^{\top} .
$$

Further, we can assume w.l.o.g. that $r=0$, or $x=0$ for $\mathbf{q}(u, v)$ lying in a space-, or time-like hyperplane, respectively. In the former case we obtain

$$
\mathbf{n}^{ \pm}=\left(\mp \sigma\left(\hat{\mathbf{a}}_{u} \times \hat{\mathbf{a}}_{v}\right), \hat{C}\right)^{\top}
$$

and similarly for the latter case. Thus, in both hyperplanar cases $\mathbf{n}^{+}$is constant if and only if $\mathbf{n}^{-}$is constant.

\section{ENVELOPE COMPUTATION}

We describe a symbolic-numerical algorithm for computing a rational parameterization of the envelope of a 2-parameter family of spheres given by a quadratic patch (11) over $\triangle$. We assume that $C \geq 0$ over $\triangle$, i.e., all associated tangent planes are space- or light-like. The envelope construction is based on the computation of an adapted parameterization of the absolute quadric $\Sigma$, which is then substituted in the reparameterization formula (16).

\subsection{Subdividing the parameter domain}

This procedure subdivides a quadratic Bézier triangle $A$ given by $\mathbf{a}(u, v)$ so that the interiors of the parameter domains of subpatches $A_{i}$ do not contain any exceptional lines of $A$, which cause singularities in the isotropic Gauss image. After the subdivision, each subpatch $A_{i}$ is reparameterized such that its parameter domain is again the standard triangle $\triangle$.

Recall that the set $\mathcal{K}$ of all exceptional lines of $A$ consists of at most 6 straight lines in the parameter domain. The set $\mathcal{K}$ can be determined by solving the polynomial equation $P(u, v)=0$ obtained by eliminating $n_{1}, n_{2}, n_{3}, n_{4}$ (e.g. using Gröbner bases) from the system of equations (12) and (17) and setting $n_{4}=1$. We consider two cases:

1. $\mathcal{K} \cap \operatorname{int}(\triangle)=\emptyset$. As no line of $\mathcal{K}$ intersects $\operatorname{int}(\triangle)$ in this case, no subdivision is required.

2. $\mathcal{K} \cap \operatorname{int}(\triangle) \neq \emptyset$. In this case we subdivide the domain $\triangle$ along the lines of $\mathcal{K}$ which intersect $\operatorname{int}(\triangle)$. The resulting triangulation of $\triangle$ has to satisfy that no subtriangle contains any point of any exceptional line as an inner point.

For further steps, we have to distinguish between exact and numerical subdivision:

Exact subdivision. Hyperplanar patches and patches belonging to classes (i)-(iv), cf. Table 1, can be, if necessary, subdivided exactly along their exceptional lines, since these lines can be computed by solving the equation $P(u, v)$ of degree at most 4 (see Table 1, Remark 2 and [2]). In other words, one can easily decide whether $\mathcal{K} \cap \operatorname{int}(\triangle)=\emptyset$ for these patches. 

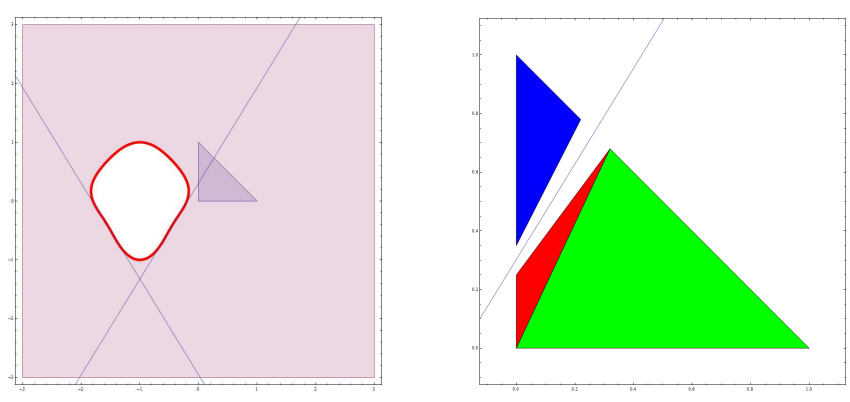

Figure 1: Left: One of the exceptional lines (thin blue) intersecting $\triangle$; Right: A numerical subdivision of $\triangle$ using Bézier clipping.
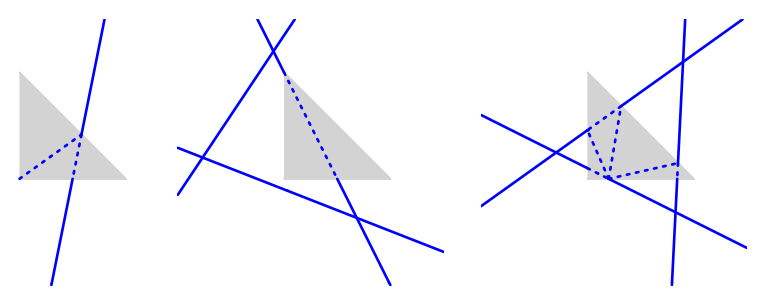

Figure 2: Several typical mutual positions of $\mathcal{K}$ (blue lines) and $\triangle$ (gray triangles). A suitable triangulation of $\triangle$ is indicated by the dashed line segments.

Numerical subdivision. For patches of class (v), the exceptional lines can be computed by solving the polynomial equation $P(u, v)=0$ of total degree 6 in $u$, $v$, which is not generally solvable in radicals. Nevertheless, it is not necessary to know the exact equations of lines in $\mathcal{K}$ to determine whether $\mathcal{K} \cap \operatorname{int}(\triangle)=\emptyset$. It is enough to study the number of real roots of the univariate polynomials $P(u, 0), P(0, v)$, $P(u, 1-u)$ on the interval $(0,1)$, i.e., the intersections of $\mathcal{K}$ with the boundary of $\triangle$. This consideration is based on the Sturm's theorem and Sturm sequences (cf. [15]). If there are no intersections, no subdivision is required.

A more complicated situation occurs for $\mathcal{K} \cap \operatorname{int}(\triangle) \neq \emptyset$ when the exceptional lines in $\mathcal{K}$ cannot be computed exactly. In this case we use Bézier clipping, see [27], or any other suitable numerical polynomial solver, to determine fat lines of width $\varrho$ (or a region bounded by two, not necessarily parallel, straight lines and the sides of $\triangle$ ) within which the exceptional lines restricted to $\triangle$ lie, see Fig. 1 . This can be achieved with any prescribed level of accuracy. Then, the triangular subdivision of $\triangle$ is adapted in such a way that the boundaries of all the fat lines that intersect $\triangle$ do not lie in the interiors of any resulting subtriangles. See Fig. 1 and Fig. 2 for examples of several such subdivisions.

\subsection{Covering the isotropic Gauss image}

For each patch obtained in Step 1, we find the two components of the isotropic Gauss image $\mathcal{G}^{ \pm}$restricted to $\triangle$ and cover one of them by a rational quadratic patch $\mathbf{r}(s, t)$ (see Fig. 5) using a stereographic projection. In order to keep the notation simple, we denote with $A$ and $\mathbf{a}(u, v)$ each subpatch created by Step 1 and its quadratic parameterization, respectively.
Depending on the output of the first part, we have to distinguish three cases:

1. The set $\mathcal{K}$ does not contain any side of $\triangle$. In this case, both $\mathcal{G}^{+}$and $\mathcal{G}^{-}$are triangles on $\Sigma$, which are bounded by the isotropic normal vectors along the patch boundary - see Fig. 3 (left).

2. $A$ is a non-hyperplanar patch and $\mathcal{K}$ contains one side of $\triangle$. Hence, exactly one of $\mathcal{G}^{+}$, or $\mathcal{G}^{-}$(see Lemma 5 ) is a biangle on $\Sigma$, bounded by the isotropic normal vectors along the two patch boundaries corresponding to the non-exceptional lines in the parameter domain - see Fig. 3 (middle).

3. $A$ is a patch lying in a space- or time-like hyperplane and $\mathcal{K}$ contains one side of $\triangle$. Hence, both $\mathcal{G}^{+}$and $\mathcal{G}^{-}$ are biangles on $\Sigma$, see Fig. 3 (right).

Taking only one part of the isotropic Gauss image (preferably we choose a triangular part, if it exists - w.l.o.g. $\mathcal{G}^{+}$) allows us to apply the methods which were originally constructed for quadratic patches in the Euclidean space $\mathbb{R}^{3}$ in [2]. For the sake of brevity, we recall only the main steps.

We use the stereographic projection $\sigma_{\mathbf{z}}$ with the pole $\mathbf{z}$ mapping the points of $\Sigma$ into the plane $\pi_{\mathbf{z}}: \mathbf{z} \cdot \mathbf{x}=0$. The image $\Omega=\sigma_{\mathbf{z}}\left(\mathcal{G}^{+}\right)$is either a curved triangle for triangular $\mathcal{G}^{+}$, or a curved biangle for biangular $\mathcal{G}^{+}$. Next, we choose a bounding triangle $\tilde{\Omega} \supset \Omega$ with straight line boundaries. We describe $\tilde{\Omega}$ as a linear Bézier patch and using the inverse stereographic projection we obtain the quadratic patch $R$ on $\Sigma$ which covers $\mathcal{G}^{+}$.

\subsection{Parameterizing the envelope and trim- ming}

We consider a patch $A$ with the quadratic parameterization $\mathbf{a}(u, v)$ representing a medial surface transform and the corresponding covering patch $R$ with the quadratic rational parameterization $\mathbf{r}(s, t)$. We compute the rational parametric representations $\mathbf{b}^{ \pm}$of the two branches of the envelope associated to the patch $\mathbf{a}(u, v)$. We also identify the exact parameter domain $D_{\mathrm{b}}$.

First step: Envelope. The rational quadratic patch $R$ obtained in Step 2 can be written in the form

$$
\mathbf{r}(s, t)=\left(r_{1}(s, t), r_{2}(s, t), r_{3}(s, t), r_{4}(s, t)\right)^{\top} .
$$

Substituting $\mathbf{r}(s, t)$ for $\mathbf{n}$ in (16) we arrive at the reparameterization

$$
\phi: u_{\mathbf{b}}=\frac{\left|\begin{array}{ll}
\left\langle\mathbf{r}, \mathbf{a}_{11}\right\rangle & \left\langle\mathbf{r}, \mathbf{a}_{10}\right\rangle \\
\left\langle\mathbf{r}, \mathbf{a}_{02}\right\rangle & \left\langle\mathbf{r}, \mathbf{a}_{01}\right\rangle
\end{array}\right|}{\left|\begin{array}{ll}
\left\langle\mathbf{r}, \mathbf{a}_{20}\right\rangle & \left\langle\mathbf{r}, \mathbf{a}_{11}\right\rangle \\
\left\langle\mathbf{r}, \mathbf{a}_{11}\right\rangle & \left\langle\mathbf{r}, \mathbf{a}_{02}\right\rangle
\end{array}\right|}, v_{\mathbf{b}}=\frac{\left|\begin{array}{ll}
\left\langle\mathbf{r}, \mathbf{a}_{10}\right\rangle & \left\langle\mathbf{r}, \mathbf{a}_{20}\right\rangle \\
\left\langle\mathbf{r}, \mathbf{a}_{01}\right\rangle & \left\langle\mathbf{r}, \mathbf{a}_{11}\right\rangle
\end{array}\right|}{\left|\begin{array}{ll}
\left\langle\mathbf{r}, \mathbf{a}_{20}\right\rangle & \left\langle\mathbf{r}, \mathbf{a}_{11}\right\rangle \\
\left\langle\mathbf{r}, \mathbf{a}_{11}\right\rangle & \left\langle\mathbf{r}, \mathbf{a}_{02}\right\rangle
\end{array}\right|} .
$$

Then the rational parameterizations of the two branches of the envelope surface $B$ are

$$
\mathbf{b}^{ \pm}(s, t)=\hat{\mathbf{a}}(s, t)+\frac{r}{\hat{C}}\left(\mathbf{w} \pm \sqrt{C} \cdot\left(\hat{\mathbf{a}}_{s} \times \hat{\mathbf{a}}_{t}\right)\right) .
$$

Considering the degrees of $A$ and $R$, this approach gives a rational triangular patch of degree at most 10 . 

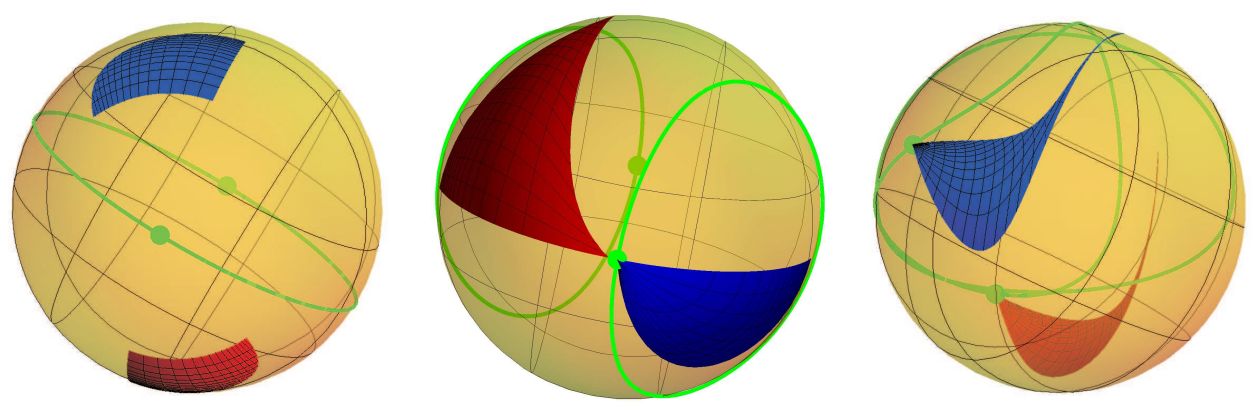

Figure 3: The two components of the isotropic Gauss image (red and blue) of a triangular quadratic patch $\mathbf{a}(u, v)$. Left: two triangles, Middle: a triangle and a biangle, Right: two biangles.

Second step: Trimming. Now, we are to find the exact parameter domain for the envelope surface. Since the patch $R$, which covers the isotropic Gauss image $\mathcal{G}^{+}$, is generally "bigger" than $\mathcal{G}^{+}$, it may also contain points with isotropic normal vectors which do not correspond to isotropic normal vectors of the patch given by a over $\triangle$. Hence, the branches of the envelope surface $B$ in (23) over $\triangle$ are also bigger than the exact branches of the envelope surface, and we need to restrict the parameter domain to an appropriate subset $D_{\mathbf{b}}$.

The boundary of $D_{\mathbf{b}}$ is closely related to the reparameterization (22). It is sufficient to consider the three rational functions

$$
\begin{aligned}
u_{\mathbf{b}}=0 & =g_{1}(s, t), \\
v_{\mathbf{b}}=0 & =g_{2}(s, t), \\
1-u_{\mathbf{b}}-v_{\mathbf{b}}=0 & =g_{3}(s, t)
\end{aligned}
$$

As $u=0, v=0$ and $u+v-1=0$ determine the boundary of $\triangle$ for $\mathbf{a}(u, v), g_{j}(s, t)$ in (24) correspond to images of this boundary under the reparameterization (22). Hence, the new parameter domain $D_{\mathbf{b}}$ is a restriction of $\triangle$ with the boundary given by (24), i.e.,

$$
D_{\mathbf{b}}=\left\{(s, t) \in \Delta: \bigcap_{j=1}^{3} g_{j}(s, t) \geq 0\right\} .
$$

Third step: Estimation of the error. For all the subpatches whose domains were computed exactly, i.e., without using Bézier clipping, we skip this step as the previous one, the trimming, yields an exact domain for the envelope patch.

On the other hand, the envelope subpatches whose parameterizations were computed with the help of Bézier clipping do not have their parameter domains trimmed exactly. In fact, the collection of subpatches does not cover the sought envelope entirely and there are "gaps" between those envelope subpatches. However, these gaps can be made as small as prescribed, e.g. by the resolution limit of a cutting tool.

In order to avoid numerical instabilities at the boundary and at singular points, we assume that there exists a real positive constant $\epsilon$ such that $C \geq \epsilon$.
LEMma 6. Consider the polynomial function, curve or surface of degree $n$ given by

$$
\mathbf{a}(u, v)=\sum_{i=0}^{n-j} \sum_{j=0}^{n} \mathbf{a}_{i, j} u^{i} v^{j} .
$$

Let $M=\max \left\{\left\|\mathbf{a}_{i, j}\right\| ; i=0, \ldots, n-j, j=0, \ldots, n\right\}, \alpha \in$ $[0,2 \pi), U, V, \varrho \in[0,1]$ and $\bar{U}=U+\varrho \cos \alpha, \bar{V}=V+\varrho \sin \alpha$. Then

$$
\begin{aligned}
\|\mathbf{a}(\bar{U}, \bar{V})-\mathbf{a}(U, V)\| & \leq M \cdot \varrho \cdot n \cdot \frac{4 \cdot 2^{n}-n-3}{2}, \\
\|\mathbf{a}(U, V)\| & \leq M \frac{(n+1)(n+2)}{2}
\end{aligned}
$$

and

$$
\|\mathbf{a}(\bar{U}, \bar{V})\| \leq M\left(1+n \cdot 2^{n+1}\right) .
$$

Proof. Changing the summation in $\mathbf{a}(u, v)$ to a diagonal one and observing that $\mathbf{a}_{0,0}$ cancels out, we have

$$
\begin{aligned}
& \|\mathbf{a}(\bar{U}, \bar{V})-\mathbf{a}(U, V)\| \leq \\
& \left\|\sum_{i=0}^{j} \sum_{j=0}^{n} \mathbf{a}_{i, j-i} \bar{U}^{i} \bar{V}^{j-i}-\mathbf{a}_{i, j-i} U^{i} V^{j-i}\right\|= \\
& \left\|\sum_{i=0}^{j} \sum_{j=1}^{n} \mathbf{a}_{i, j-i} p\right\|,
\end{aligned}
$$

where

$$
p=p(\varrho, \alpha, U, V, i, j)=\bar{U}^{i} \bar{V}^{j-i}-U^{i} V^{j-i} .
$$

Expanding $p$ and rearranging the terms, we arrive at

$$
\begin{aligned}
& p+U^{i} V^{j-i}= \\
& \left(\sum_{k=0}^{i}\left(\begin{array}{c}
i \\
k
\end{array}\right) U^{i-k} \varrho^{k} \cos ^{k} \alpha\right)\left(\sum_{l=0}^{j-i}\left(\begin{array}{c}
j-i \\
l
\end{array}\right) V^{j-i-l} \varrho^{l} \sin ^{l} \alpha\right)= \\
& \sum_{k=0}^{i} \sum_{l=0}^{j-i}\left(\begin{array}{c}
i \\
k
\end{array}\right) U^{i-k} \varrho^{k} \cos ^{k} \alpha\left(\begin{array}{c}
j-i \\
l
\end{array}\right) V^{j-i-l} \varrho^{l} \sin ^{l} \alpha= \\
& \sum_{k=0}^{l} \sum_{l=0}^{j}\left(\begin{array}{c}
i \\
k
\end{array}\right)\left(\begin{array}{c}
j-i \\
l-k
\end{array}\right) U^{i-k} V^{j-i-l+k} \varrho^{l} \cos ^{k} \alpha \sin ^{l-k} \alpha .
\end{aligned}
$$

As the term for which $k=l=0$ cancels out with $U^{i} V^{j-i}$, we see that

$$
\begin{aligned}
& p=\sum_{k=0}^{l} \sum_{l=1}^{j}\left(\begin{array}{c}
i \\
k
\end{array}\right)\left(\begin{array}{l}
j-i \\
l-k
\end{array}\right) V^{j-i-l+k} U^{i-k} \varrho^{l} \cos ^{k} \alpha \sin ^{l-k} \alpha= \\
& \varrho \sum_{k=0}^{l} \sum_{l=1}^{j}\left(\begin{array}{l}
i \\
k
\end{array}\right)\left(\begin{array}{l}
j-i \\
l-k
\end{array}\right) V^{j-i-l+k} U^{i-k} \varrho^{l-1} \cos ^{k} \alpha \sin ^{l-k} \alpha .
\end{aligned}
$$

Consequently

$$
|p| \leq \varrho \sum_{k=0}^{l} \sum_{l=1}^{j}\left(\begin{array}{l}
i \\
k
\end{array}\right)\left(\begin{array}{l}
j-i \\
l-k
\end{array}\right)=\varrho\left(2^{j}-1\right) .
$$


Summing up, we conclude that

$$
\begin{aligned}
& \|\mathbf{a}(\bar{U}, \bar{V})-\mathbf{a}(U, V)\| \leq \sum_{i=0}^{j} \sum_{j=1}^{n}|p| \cdot\left\|\mathbf{a}_{i, j-i}\right\| \leq \\
& M \sum_{i=0}^{j} \sum_{j=1}^{n}|p| \leq M \cdot \varrho \cdot \sum_{i=0}^{j} \sum_{j=1}^{n}\left(2^{j}-1\right)= \\
& M \cdot \varrho \cdot n \cdot \frac{4 \cdot 2^{n}-n-3}{2} .
\end{aligned}
$$

The two upper bounds (28) and (29) follow from the computations above.

Now, we can formulate:

THEOREM 7. Let $\mathbf{b}^{ \pm}(u, v)$ be the envelope parameterization (6) corresponding to a quadratic medial surface transform $\mathbf{a}(u, v)$ for which $C \geq \epsilon$. Let $\alpha \in[0,2 \pi), U, V, \varrho \in[0,1]$ and $\bar{U}=U+\varrho \cos \alpha, \bar{V}=V+\varrho \sin \alpha$. Then

$$
\left\|\mathbf{b}^{ \pm}(\bar{U}, \bar{V})-\mathbf{b}^{ \pm}(U, V)\right\| \leq K(M, \epsilon) \cdot \varrho,
$$

where $K$ is a constant that only depends on $M=$ $\max \left\{\left\|\mathbf{a}_{i, j}\right\|\right\}$.

Proof. Let $\mathbf{s}=\hat{\mathbf{a}}_{u} \times \hat{\mathbf{a}}_{v}$. Further, let $r, \mathbf{w}, \mathbf{s}, C$ and $\hat{\mathbf{a}}$ be the quantities in the envelope formula computed using parameter values $U$ and $V$, while $\bar{r}, \overline{\mathbf{w}}, \overline{\mathbf{s}}, \bar{C}$ and $\overline{\mathbf{a}}$ (here we drop the extra ^ symbol) using parameter values $\bar{U}$ and $\bar{V}$. Recalling the envelope formula (6), one can see that

$$
\left\|\overline{\mathbf{b}}^{ \pm}-\mathbf{b}^{ \pm}\right\| \leq\|\overline{\mathbf{a}}-\mathbf{a}\|+\frac{1}{\epsilon}\|\bar{r}(\overline{\mathbf{w}} \pm \overline{\mathbf{s}} \sqrt{\bar{C}})-r(\mathbf{w} \pm \mathbf{s} \sqrt{C})\| .
$$

Using the formula

$$
|| \alpha \mathbf{a}-\beta \mathbf{b} \| \leq|\alpha-\beta| \cdot|| \mathbf{a}||+|\beta| \cdot|| \mathbf{a}-\mathbf{b}||
$$

for scalars $\alpha, \beta$ and vectors $\mathbf{a}, \mathbf{b}$ and Lemma 6 , we see that there exist constants $K_{1}, K_{2}, K_{3}$ and $K_{4}$ such that

$$
\begin{aligned}
& \left\|\overline{\mathbf{b}}^{ \pm}-\mathbf{b}^{ \pm}\right\| \leq K_{1} \varrho+\frac{1}{\epsilon}(|\bar{r}-r| \cdot\|\overline{\mathbf{w}} \pm \overline{\mathbf{s}} \sqrt{\bar{C}}\|)+ \\
& \frac{|r|}{\epsilon}(\|\overline{\mathbf{w}}-\mathbf{w}\|+\|\overline{\mathbf{s}} \sqrt{\bar{C}}-\mathbf{s} \sqrt{C}\|) \leq \\
& K_{1} \varrho+\frac{1}{\epsilon}\left(K_{2} \varrho(\|\overline{\mathbf{w}}\|+\sqrt{\bar{C}}|| \overline{\mathbf{s}} \|)\right)+ \\
& \left.\frac{K_{3}}{\epsilon}\left(K_{4} \varrho+|\sqrt{\bar{C}}-\sqrt{C}| \cdot\|\overline{\mathbf{s}}\|+\sqrt{C} \cdot\|\overline{\mathbf{s}}-\mathbf{s}\|\right)\right) .
\end{aligned}
$$

Further, observe that there exists a constant $K_{5}$ such that

$$
|\sqrt{\bar{C}}-\sqrt{C}|=\frac{|\bar{C}-C|}{\sqrt{\bar{C}}+\sqrt{C}} \leq \frac{K_{5} \varrho}{2 \epsilon} .
$$

By Lemma 6 , there exist constants $K_{6}, K_{7}, K_{8}$ and $K_{9}$ such that

$$
\begin{aligned}
& \left\|\overline{\mathbf{b}}^{ \pm}-\mathbf{b}^{ \pm}\right\| \leq \\
& K_{1} \varrho+\frac{1}{\epsilon}\left(K_{2} K_{6} \varrho+K_{3}\left(K_{4} \varrho+\frac{K_{5} \varrho}{2 \epsilon} K_{7}+K_{8} K_{9} \varrho\right)\right) \leq \\
& K(M, \epsilon) \cdot \varrho .
\end{aligned}
$$

In other words, anytime we half the error $\varrho$ (the width of the fat lines) in the parameter domain using Bézier clipping, the bound on the error on the envelope boundary corresponding to the image of an exceptional line on $\mathbf{a}(u, v)$ is reduced by the factor $\frac{1}{2}$. Consequently, using a sufficient number of Bézier clipping steps (i.e., $\varrho$ approaches zero), we can make the envelope boundary error arbitrarily small (e.g. below the resolution limit used in practice).
Remark 4. The offsets of the envelope, resp. their rational parameterizations, can be obtained by lifting the corresponding medial surface transform $\mathbf{a}(u, v)$ in the direction of its last coordinate. Thus, $d$-offsets correspond to the substitution $r(u, v)=r(u, v) \pm d$ in (6). In this case, Theorem 7 applies as well with a slight modification: the error bound constant $K$ depends on $d$ as well.

\section{SUMMARY}

In this section, we give a summary of the algorithm described in Section 4 and mention some of its applications.

Function 1 subdivides the quadratic patch such that the interiors of output subpatches do not contain any exceptional lines. It uses exact subdivision, if it is possible, numerical subdivision otherwise.

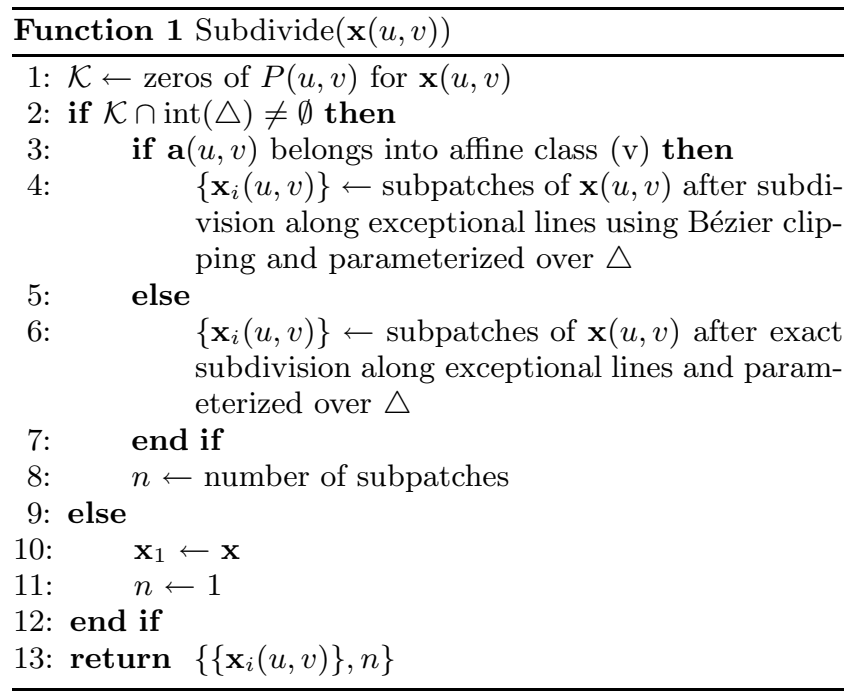

Function 2 covers each of the subpatches returned by Function 1 by a circumscribed rational Bézier patch on $\Sigma$ using stereographic and inverse stereographic projection with a suitable pole and plane.

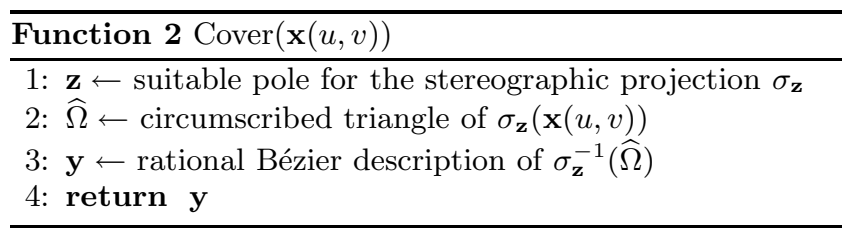

Using the circumscribing rational Bézier patch returned by Function 2, Function 3 finds the reparameterization of the input patch such that it fulfills the MOS condition. Moreover, it returns the exact parameter domain of the reparameterized patch as a subset of the domain $\triangle$.

The method presented in this paper (summarized in Algorithm 4) can serve as a first step for a range of subsequent applications. Considering general (free-form) medial surface transforms in $\mathbb{R}^{3,1}$, one can produce their $C^{1}$ approximations using quadratic Bézier patches (cf. [28, 36]). The approximating MST closely captures the structure of a given domain and the rationality of the domain boundaries is guaranteed. Furthermore, using this approach, one obtains rational approximations of the domain offsets as well. 

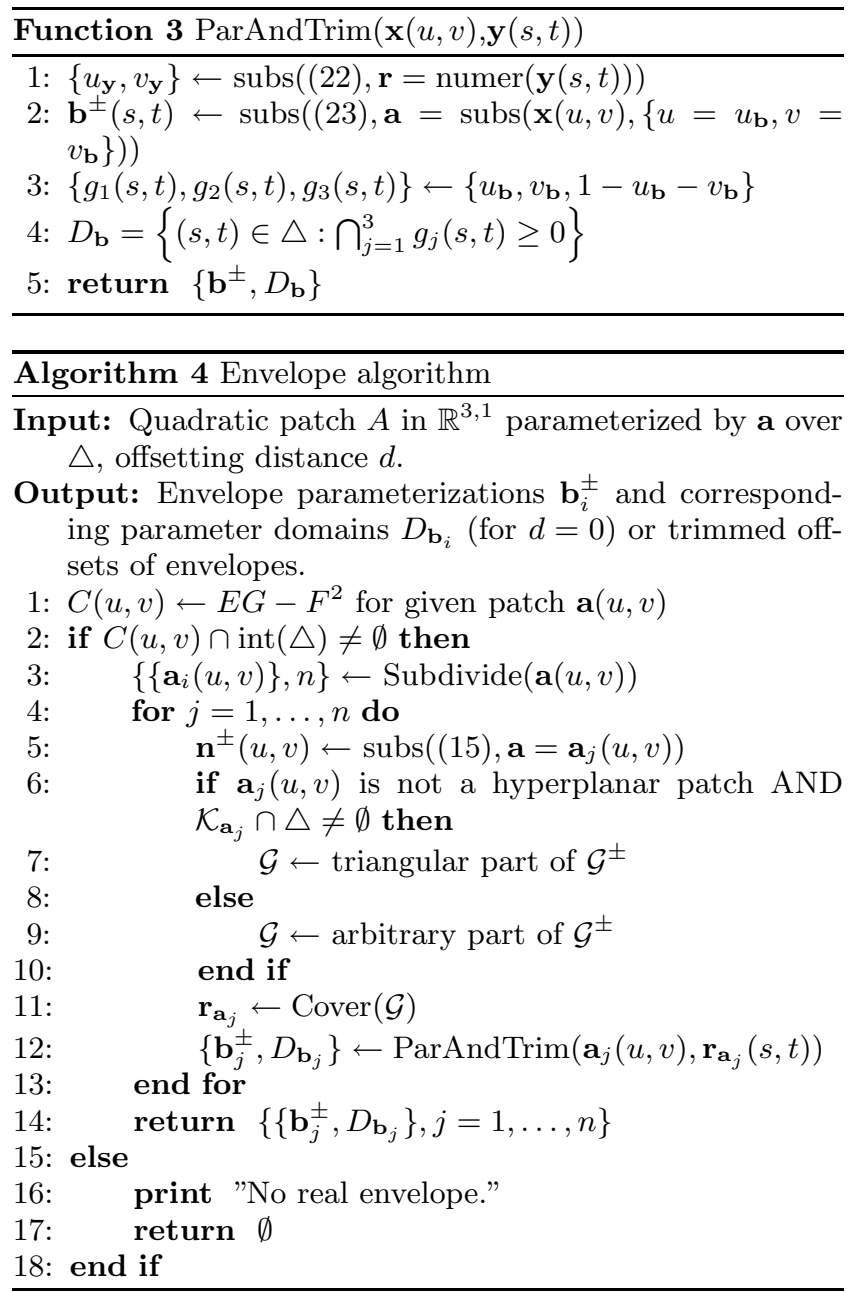

Most of the existing techniques approximate the offsets directly and the operation of trimming follows. However, using the recent results about quadratic Bézier triangles in $\mathbb{R}^{3,1}$, one may approximate the MST instead and represent the associated envelope and all its $d$-offsets exactly. Obviously, this approach simplifies the computation of selfintersections of $d$-offsets considerably since it only requires solving $r(u, v) \geq d$, where $d$ can be considered as a cutting tool radius used in practice. This inequality can be easily incorporated into the envelope algorithm by adding the extra trimming condition $g_{4}(s, t) \geq 0$ to (25), where $g_{4}(s, t)=r(u(s, t), v(s, t))-d$.

\section{EXAMPLES}

In this section we demonstrate the algorithm on several examples. Each example corresponds to one of the possible situations - Examples 1-3 cover different cases which can occur in exact subdivision, Example 4 is devoted to numerical subdivision, cf. Section 4.1.

Example 1. Consider the patch

$$
\begin{aligned}
\mathbf{a}(u, v)= & \left(\left(u+\frac{1}{10}\right)^{2},\left(v+\frac{2}{3}\right)^{2}, u+\frac{1}{10}, v+\frac{2}{3}\right)^{\top}, \\
& (u, v) \in \triangle .
\end{aligned}
$$
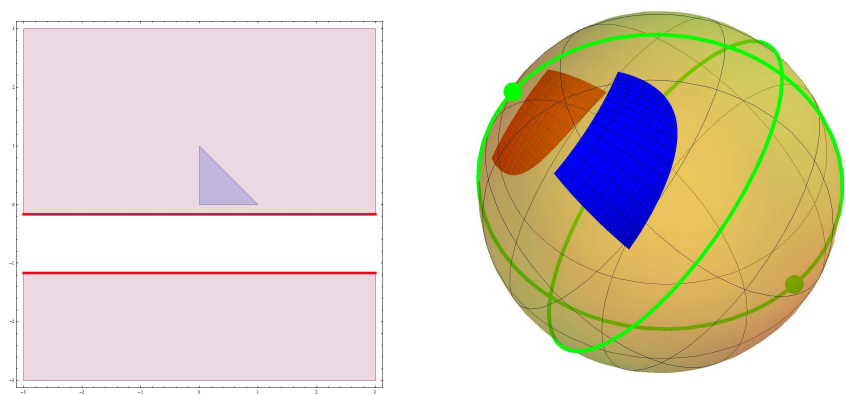

Figure 4: (Example 1) Left: The curve $C(u, v)=0$ (thick red curve), space-like domain $C(u, v)>0$ (purple), exceptional lines (thin blue curves, coincident with $C(u, v)=0$ here); Right: Isotropic Gauss image (red and blue), the curve $\mathcal{S}_{\Sigma}$ (green curve) and exceptional normals (green points).

The space-like domain of $\mathbf{a}(u, v)$ is bounded by the curve

$$
C(u, v)=\frac{2}{225}\left(50 u^{2}+10 u+13\right)(6 v+1)(6 v+7) .
$$

The parameter domain $\triangle$ of the patch lies inside the spacelike domain. The polynomial $P(u, v)$ describing exceptional lines has the form

$$
(6 v+1)(6 v+7)=0 .
$$

Thus, $\mathcal{K}$ contains two exceptional lines corresponding to the factors of (44), see Fig. 4 (left). Since $\mathcal{K} \cap \operatorname{int}(\triangle)=\emptyset$, no subdivision is required.
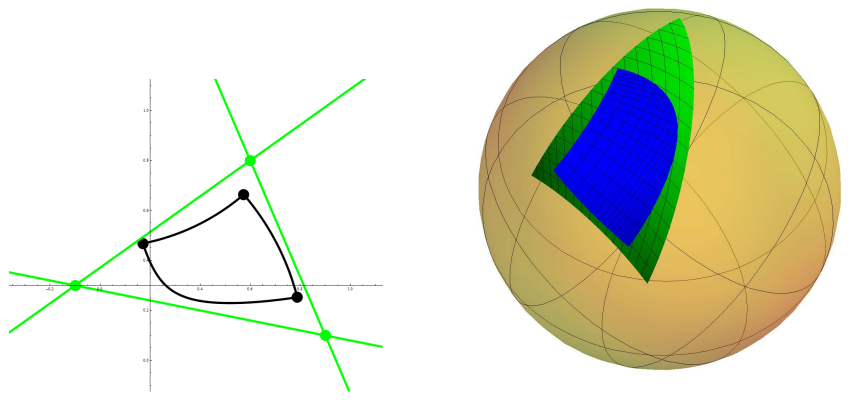

Figure 5: (Example 1) Left: Stereographic projection of $\mathcal{G}^{+}$(black) and a circumscribed triangle (green); Right: $\mathcal{G}^{+}$(blue) and its covering patch (green).

Next, we need to find the isotropic normal vectors $\mathbf{n}^{ \pm}$of $\mathbf{a}(u, v)$. Using the formula (15), we obtain

$$
\begin{aligned}
\mathbf{n}^{ \pm}(u, v)= & \left( \pm \frac{5(6 v+1)(6 v+7)}{m}, \frac{3}{6 v+4},\right. \\
& \left.\mp \frac{(10 u+1)(6 v+1)(6 v+7)}{m}\right)^{\top},
\end{aligned}
$$

where

$$
m=2 \sqrt{2}(3 v+2) \sqrt{(10 u(5 u+1)+13)(6 v+1)(6 v+7)} .
$$



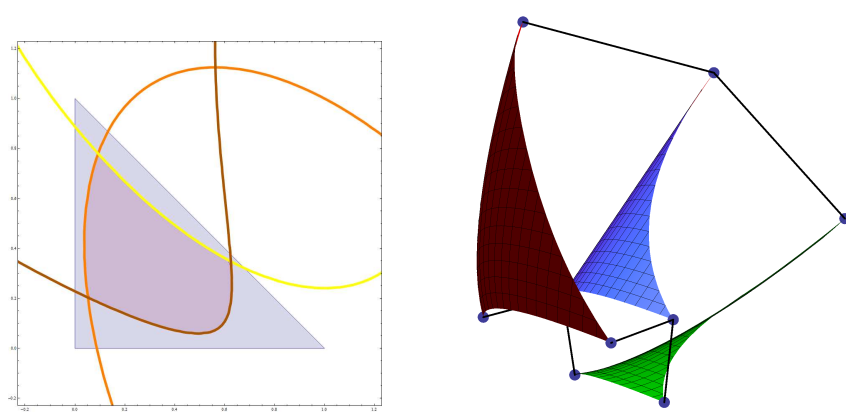

Figure 6: (Example 1) Left: Final domain $D_{\mathrm{b}}$, polynomials $g_{1}(s, t)=0$ (orange), $g_{2}(s, t)=0$ (yellow) and $g_{3}(s, t)=0$ (brown); Right: Medial surface (blue) and envelopes (red and green).

Restriction of (45) to the closure of the space-like domain provides the description of the isotropic Gauss image $\mathcal{G}^{ \pm}$for the patch $\mathbf{a}(u, v)$. We can also find the singular curve $\mathcal{S}_{\Sigma}$ and the exceptional normals $(0,1,0)$ and $(0,-1,0)$ corresponding to the exceptional lines, see Fig. 4 (right).

As mentioned in Section 4.2, we take only one component of $\mathcal{G}^{ \pm}$, e.g. we can use $\mathcal{G}^{+}$(blue region in Fig. 4 (right)). Further, we use stereographic projection $\sigma_{\mathbf{z}}$ with a suitably chosen pole $\mathbf{z}\left(\right.$ e.g. $\left.(0,0,1)^{\top}\right)$ and plane $\pi_{\mathbf{z}}(z=0)$. Then we project the boundary curves of $\mathcal{G}^{+}$to the plane $\pi_{\mathbf{z}}$ and obtain the curved triangle shown in Fig. 5 (black curves). This curved triangle is then circumscribed by a suitable triangle which is parameterized as a linear Bézier triangle. Using the inverse stereographic projection we obtain the circumscribed rational quadratic Bézier triangle $R$ on $\Sigma$ (green patch in Fig. 5), which covers $\mathcal{G}^{+}$, in the form

$$
\begin{aligned}
\mathbf{r}(s, t)= & (-10(-9+10 s+3 t), 10(1+2 s+7 t) \\
& -9+52 s^{2}+44 s(-2+t)-20 t+29 t^{2}, \\
& \left.91+52 s^{2}+44 s(-2+t)-20 t+29 t^{2}\right)^{\top} .
\end{aligned}
$$

Consequently, we substitute (46) into (22) in order to get the reparameterization

$$
\begin{aligned}
\phi:(u, v)= & \left(\frac{9+52 s^{2}-26 t+29 t^{2}+4 s(-27+11 t)}{20(-9+10 s+3 t)}, \quad\right. \text { (47) } \\
& \left.\frac{233+156 s^{2}-340 t+87 t^{2}+4 s(-86+33 t)}{60(1+2 s+7 t)}\right) .
\end{aligned}
$$

Then $\mathbf{a}(\phi(s, t))$ fulfills the MOS condition.

Finally, we have to find the new parameter domain $D_{\mathbf{b}}(25)$ for the reparameterized patch $\mathbf{a}(\phi(s, t))$. The domain $D_{\mathbf{b}}$ is shown in Fig. 6 (left), the medial surface together with the corresponding envelopes are shown in Fig. 6 (right).

\section{Example 2. Consider the patch}

$\mathbf{a}(u, v)=\left(\left(u+\frac{1}{2}\right)^{2}, v^{2},\left(u+\frac{1}{2}\right) v, u+\frac{1}{2}\right)^{\top},(u, v) \in \triangle$.

The space-like domain of $\mathbf{a}(u, v)$ is bounded by the curve

$$
C(u, v)=4 u^{4}+8 u^{3}+u^{2}\left(16 v^{2}+5\right)+16 u v^{2}+u+4 v^{4} \text {. }
$$
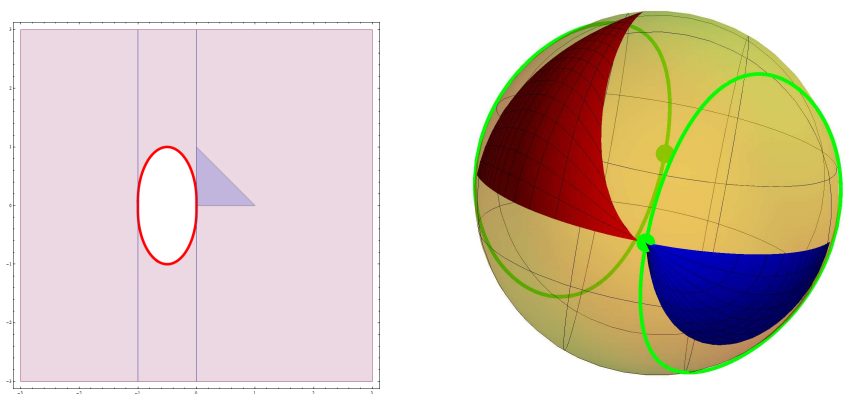

Figure 7: (Example 2) Left: The curve $C(u, v)=0$ (thick red curve), space-like domain $C(u, v)>0$ (purple), exceptional line (thin blue curve); Right: Isotropic Gauss image (red and blue), the curve $\mathcal{S}_{\Sigma}$ (green curve) and exceptional normals (green points).

The parameter domain $\triangle$ of the patch lies inside the spacelike domain. We can find the polynomial determining the exceptional lines in the form

$$
u(u+1)=0 .
$$

Thus, $\mathcal{K}$ contains two real exceptional lines corresponding to the factors $u$ and $u+1$ of (50), see Fig. 7 (left). Since $\mathcal{K} \cap \operatorname{int}(\triangle)=\emptyset$, no subdivision is needed. Nevertheless, $\mathcal{K} \cap \triangle \neq \emptyset$ and the intersection corresponds to one side of $\triangle$.

We find the isotropic normal vectors $\mathbf{n}^{ \pm}$of $\mathbf{a}(u, v)$ using (15). The restriction to the closure of the space-like domain provides the description of the isotropic Gauss image $\mathcal{G}^{ \pm}$for the patch $\mathbf{a}(u, v)$. Since one of the exceptional lines contains one side of $\triangle$ and $\mathbf{a}(u, v)$ is not a hyperplanar patch, one component of the isotropic Gauss image $\mathcal{G}^{ \pm}$is biangular and the other one is triangular, see Fig. 7 (right). Thus, we can take the triangular component of $\mathcal{G}^{ \pm}$and continue in the same way as in Example 1.

Example 3. Consider the patch

$$
\begin{aligned}
& \mathbf{a}(u, v)= \\
& \left(\frac{1}{400}\left(289\left(u^{2}+v^{2}\right)+17(17 v+32) u-187 v+99\right),\right. \\
& \frac{1}{400}\left(289\left(v^{2}-u^{2}\right)-17(17 v-8) u+323 v+109\right), \\
& \frac{1}{400}\left(289 u^{2}+289 v u+204 u-255 v-5\right), \\
& \left.\frac{1}{400}(17 u-15)(17 u-17 v+3)\right)^{\top},(u, v) \in \triangle .
\end{aligned}
$$

The space-like domain of $\mathbf{a}(u, v)$ is bounded by the curve

$$
\begin{aligned}
C(u, v)= & 250563 u^{4}-442170 u^{3}- \\
& 289(68 v(17 v-16)-719) u^{2}- \\
& 68(34 v-1)(17 v(17 v+14)+174) u- \\
& (17 v(17 v+14)+174)^{2}
\end{aligned}
$$

The parameter domain $\triangle$ of the patch lies inside the spacelike domain. The polynomial

$$
u\left(522-1088 u+578 u^{2}+714 v-578 u v+867 v^{2}\right)=0
$$

describes the exceptional lines of the patch, i.e., $\mathcal{K}$ contains one real exceptional line corresponding to the factor 

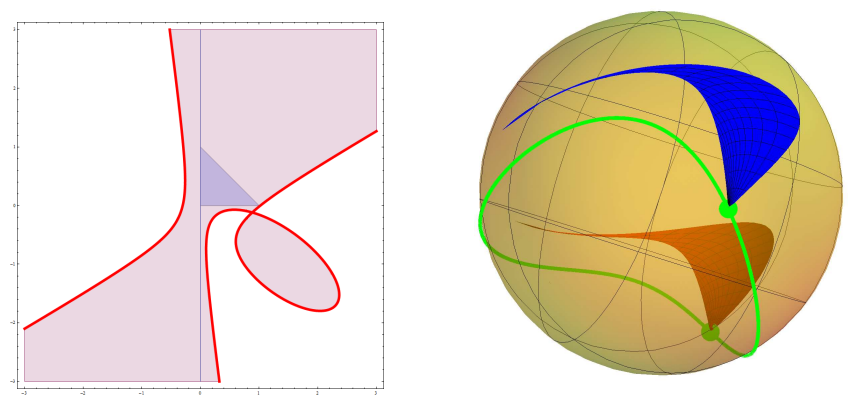

Figure 8: (Example 3) Left: The curve $C(u, v)=0$ (thick red curve), space-like domain $C(u, v)>0$ (purple), exceptional line (thin blue curve); Right: Isotropic Gauss image (red and blue), the curve $\mathcal{S}_{\Sigma}$ (green curve) and exceptional normals (green points).

$u$ of (53), see Fig. 8 (left). As in the previous example, $\mathcal{K} \cap \operatorname{int}(\triangle)=\emptyset$, but $\mathcal{K} \cap \triangle \neq \emptyset$ and the intersection corresponds to one side of $\triangle$.
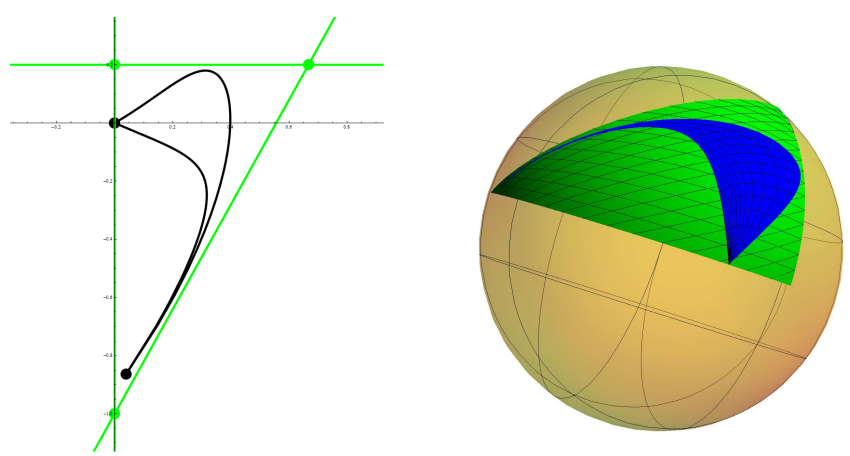

Figure 9: (Example 3) Left: Stereographic projection of $\mathcal{G}^{+}$(black) and a circumscribed triangle (green); Right: $\mathcal{G}^{+}$(blue) and its covering patch (green).

The isotropic normal vectors $\mathbf{n}^{ \pm}$of $\mathbf{a}(u, v)$ can be found by (15). Since the exceptional line contains one side of $\triangle$ and $\mathbf{a}(u, v)$ is a hyperplanar patch, both components of the isotropic Gauss image $\mathcal{G}^{ \pm}$are biangular in this case. We can also find the singular curve $\mathcal{S}_{\Sigma}$ and the exceptional normals $(0,0,-1)^{\top}$ and $(-2 / 3,2 / 3,1 / 3)^{\top}$ corresponding to the exceptional line, see Fig. 8 (right).

Similarly, we take only one component of $\mathcal{G}^{ \pm}$, e.g. we can use $\mathcal{G}^{+}$(blue region in Fig. 8). Further, we use stereographic projection $\sigma_{\mathbf{z}}$ with a suitably chosen pole $\mathbf{z}$ (e.g. $\left.(0,0,1)^{\top}\right)$ and the plane $\pi_{\mathbf{z}}(z=0)$. Then we project the boundary curves of $\mathcal{G}^{+}$to the plane $\pi_{\mathbf{z}}$ and obtain the curved biangle shown in Fig. 9 (black curves). This curved biangle is then circumscribed by a suitable triangle which is parameterized as a linear Bézier triangle. Using the inverse stereographic projection we obtain the circumscribed rational quadratic Bézier triangle $R$ on $\Sigma$ (green patch in Figure 9), which
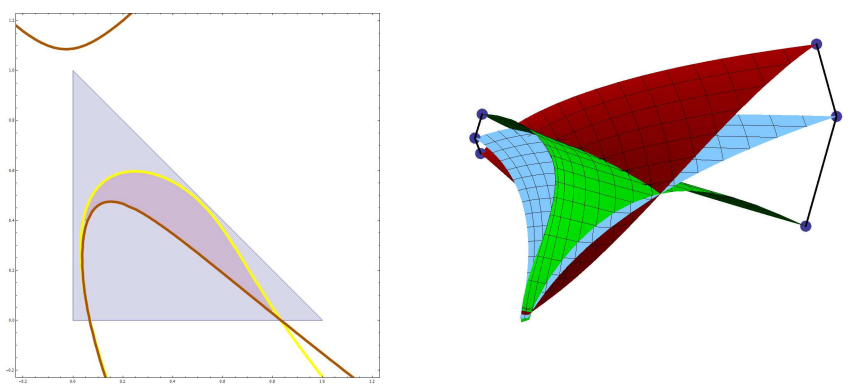

Figure 10: (Example 3) Left: Final domain $D_{\mathrm{b}}$, polynomials $g_{2}(s, t)=0$ (yellow) and $g_{3}(s, t)=0$ (brown); Right: Medial surface (blue) and envelopes (red and green).

covers $\mathcal{G}^{+}$, in the form

$$
\begin{aligned}
\mathbf{r}(s, t)= & (150 t, 45(-5+6 s+6 t), \\
& 162 s^{2}+54 s(-5+6 t)+2 t(-135+106 t), \\
& \left.225-270 s+162 s^{2}-270 t+324 s t+212 t^{2}\right)^{\top} .
\end{aligned}
$$

Consequently, we substitute (54) into (22) in order to obtain the reparameterization $\phi$. Then $\mathbf{a}(\phi(s, t))$ fulfills the MOS condition.

Finally, we have to find the new parameter domain $D_{\mathbf{b}}(25)$ for the reparameterized patch $\mathbf{a}(\phi(s, t))$. The domain $D_{\mathbf{b}}$ is shown in Fig. 10 (left), the medial surface together with the corresponding envelopes are shown in Fig. 10 (right).

\section{Example 4. Consider the patch}

$$
\mathbf{a}(u, v)=\left((u+1)^{2}+v, v^{2},(u+1) v, u+1\right)^{\top},(u, v) \in \triangle .
$$

The space-like domain of $\mathbf{a}(u, v)$ is bounded by the curve

$$
\begin{aligned}
C(u, v)= & \left(4 u^{2}+8 u+v^{2}+3\right)\left((u+1)^{2}+4 v^{2}+1\right)- \\
& (u+1)^{2}(v+2)^{2} .
\end{aligned}
$$

The parameter domain $\triangle$ of the patch lies inside the spacelike domain. The set $\mathcal{K}$ containing exceptional lines is described by the polynomial

$$
\begin{aligned}
& 64 u^{6}+384 u^{5}+256 v^{2} u^{4}-192 v u^{4}+944 u^{4}+ \\
& 1024 v^{2} u^{3}-768 v u^{3}+1216 u^{3}+64 v^{4} u^{2}- \\
& -512 v^{3} u^{2}+1616 v^{2} u^{2}-1120 v u^{2}+848 u^{2}+ \\
& 128 v^{4} u-1024 v^{3} u+1184 v^{2} u-704 v u+ \\
& +288 u-64 v^{6}-64 v^{5}+176 v^{4}-464 v^{3}+264 v^{2}- \\
& 144 v+31=0,
\end{aligned}
$$

see Fig. 11 (left). We can also compute the isotropic normal vectors $\mathbf{n}^{ \pm}$using (15) and the restriction to the closure of the space-like domain gives the description of the isotropic Gauss image $\mathcal{G}^{ \pm}$which is, together with the singular curve $\mathcal{S}_{\Sigma}$ and exceptional normals, shown in Fig. 11 (right).

As seen from Fig. 11 (left), $\mathcal{K} \cap \operatorname{int}(\triangle) \neq \emptyset$. This can be also rigorously tested by counting the numbers of roots of polynomials $P(u, 0), P(0, v)$ and $P(u, 1-u)$ in the interval $(0,1)$ using the Sturm sequences, where we obtain 0,1 and 1 

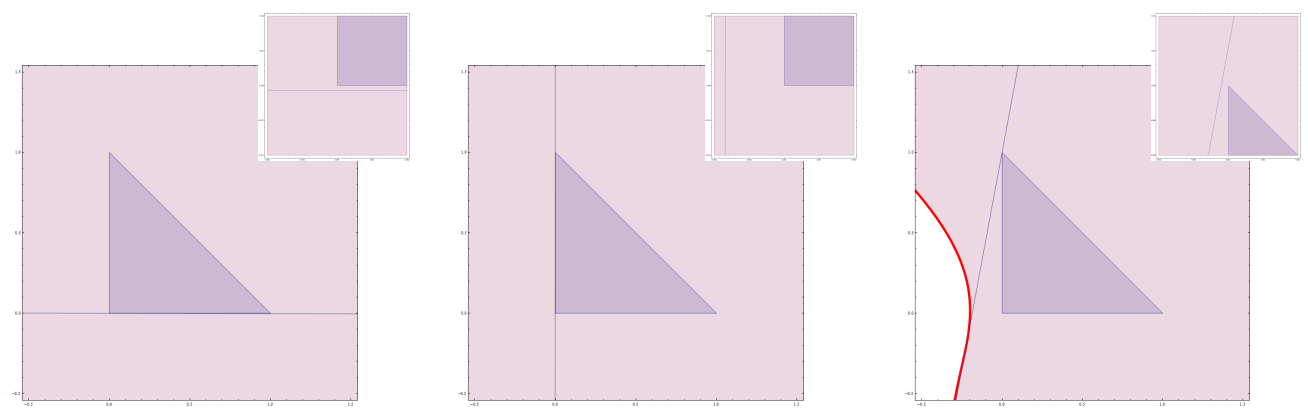

Figure 13: (Example 4) Space-like domains (purple) and exceptional lines for new patches $\mathbf{a}_{1}(u, v)$, $\mathbf{a}_{2}(u, v)$ and $\mathbf{a}_{3}(u, v)$.
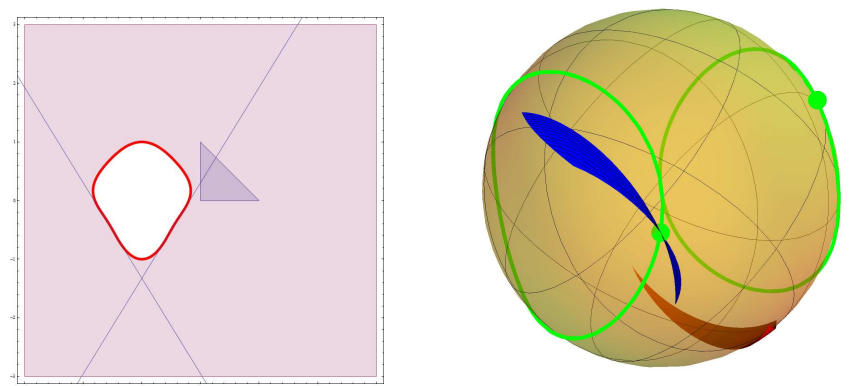

Figure 11: (Example 4) Left: The curve $C(u, v)=0$ (thick red curve), space-like domain $C(u, v)>0$ (purple), exceptional lines (thin blue curves); Right: Isotropic Gauss image (red and blue), the curve $\mathcal{S}_{\Sigma}$ (green curve) and exceptional normals (green points).

real root for these polynomials, respectively. Thus, we have to subdivide the domain along the exceptional line.

Since $\mathbf{a}(u, v)$ belongs into the affine class (v) (cf. Table 1), $P(u, v)$ is a polynomial of degree 6 which is not factorizable in radicals and we have to use the numerical approach. We choose the accuracy $\epsilon=10^{-3}$ and using the Bézier clipping we find the roots of polynomials $P(0, v)$ and $P(u, 1-u)$ in the interval $(0,1)$ with the given accuracy. Thus, we obtain intervals

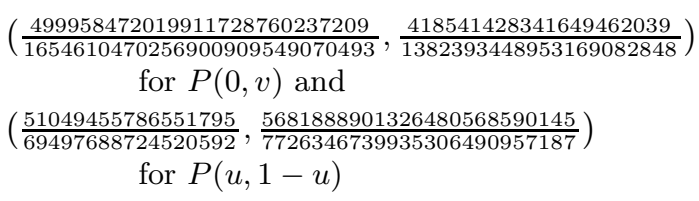

of length less than $\epsilon$ which contain the roots, i.e., the maximum width of the corresponding fat line is $\epsilon$. The boundary values of these intervals are then used to subdivide the domain $\triangle$ (see Fig. 12 (left)). For each new triangle we find the affine transformation which transforms this triangle to the standard triangle $\triangle$ and we apply this transformations also to the given patch $\mathbf{a}(u, v)$. Hence, we obtain three new patches $\mathbf{a}_{i}(u, v), i=1,2,3$, with parameter domains inside the corresponding space-like domains - see Fig. 12 (right) for their isotropic Gauss images. Observe in Fig. 13 that
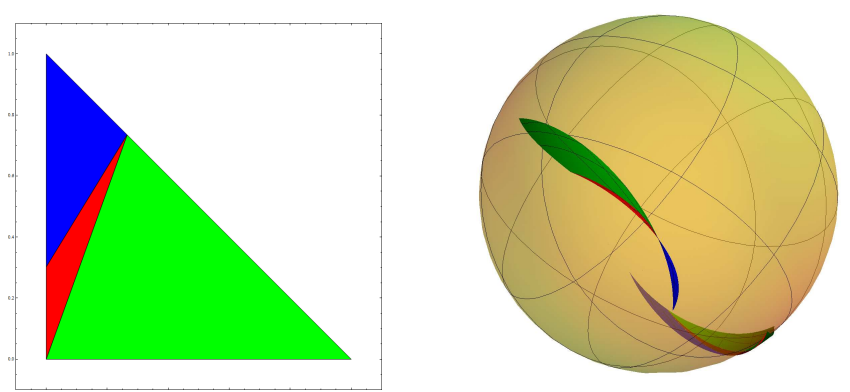

Figure 12: (Example 4) Left: The domain $\triangle$ after subdivision using the exceptional line; Right: Isotropic Gauss image of the new subpatches $\mathbf{a}_{1}(u, v)$, $\mathbf{a}_{2}(u, v)$ and $\mathbf{a}_{3}(u, v)$ (blue, red and green).

exceptional lines pass very close but outside $\triangle$. Since for all subpatches $\mathbf{a}_{i}(u, v)$ it holds $\mathcal{K} \cap \triangle=\emptyset$, we can apply the method from Example 1 on all $\mathbf{a}_{i}(u, v)$.

Finally, the error on the envelope due to the numerical subdivision, i.e., the maximal width of the gaps between subpatches of the envelope boundary corresponding to patches $\mathbf{a}_{i}(u, v)$, is less than $3.5 \epsilon$ (computed using parametric distance estimation) for both branches of the envelope boundary.

\section{ACKNOWLEDGMENTS}

B. Bastl and M. Lávička were supported by Research Plan MSM 4977751301. The authors wish to thank B. Jüttler for his valuable suggestions and assistance with the presented research. The authors would like to thank the anonymous referees for their comments.

\section{REFERENCES}

[1] O. Aichholzer, W. Aigner, F. Aurenhammer, T. Hackl, B. Jüttler, and M. Rabl: Medial axis computation for planar free-form shapes. To appear in Computer-Aided Design.

[2] B. Bastl, B. Jüttler, J. Kosinka, and M. Lávička: Computing exact rational offsets of quadratic triangular Bézier surface patches. Computer-Aided Design, Vol. 40, pp. 197-209, Elsevier, 2008. 
[3] B. Bastl, B. Jüttler, J. Kosinka, and M. Lávička: Volumes with Piecewise Quadratic Medial Surface Transforms: Computation of Boundaries and Trimmed Offsets. In preparation.

[4] H. Blum: A transformation for extracting new descriptors of shape. In: W. Wathen-Dunn (Ed.), Models for the perception of speech and visual form, pp. 362-380, MIT Press, 1967.

[5] L. Cao, Z. Jia, and J. Liu: Computation of medial axis and offset curves of curved boundaries in planar domains based on the Cesaro's approach. To appear in Computer-Aided Design.

[6] L. Cao and J. Liu: Computation of medial axis and offset curves of curved boundaries in planar domain. Computer-Aided Design, Vol. 40, pp. 465-475, Elsevier, 2008.

[7] H.Ch. Cho, H.I. Choi, S.-H. Kwon, D.S. Lee, and N.-S. Wee: Clifford algebra, Lorentzian geometry and rational parametrization of canal surfaces. Computer Aided Geometric Design, Vol. 21 , pp. 327-339, Elsevier, 2004

[8] H.I. Choi, S.W. Choi, and H.P. Moon: Mathematical theory of medial axis transform. Pacific Journal of Mathematics, Vol. 181, pp. 57-88, 1997.

[9] H.I. Choi, Ch.Y. Han, H.P. Moon, K.H. Roh, and N.-S. Wee: Medial axis transform and offset curves by Minkowski Pythagorean hodograph curves. Computer-Aided Design, Vol. 31, pp. 59-72, 1999.

[10] H.I. Choi, D.S. Lee, and H.P. Moon: Clifford algebra, spin representation and rational parameterization of curves and surfaces. Advances in Computational Mathematics, Vol. 17, pp. 5-48, 2002.

[11] W.L.F. Degen: Exploiting curvatures to compute the medial axis for domains with smooth boundary. Computer Aided Geometric Design, Vol. 21, pp. 641-660, Elsevier, 2004.

[12] G. Farin, J. Hoschek, and M.S. Kim (Eds.): Handbook of Computer Aided Geometric Design. Elsevier, 2002.

[13] R.T. Farouki, T. Sakkalis: Pythagorean hodographs. IBM Journal of Research and Development, Vol. 34, pp. 736-752, 1990.

[14] R.T.: Farouki, Pythagorean-Hodograph Curves: Algebra and Geometry Inseparable., Geometry and Computing. Springer-Verlag, 2008.

[15] D.G. Hook, P.R. McAree: Using Sturm Sequences To Bracket Real Roots of Polynomial Equations. In: A. Glassner (Ed.), Graphic Gems I, pp. 416-422, Academic Press, 1990.

[16] G.-I. Kim and M.-H. Ahn: $C^{1}$ Hermite interpolation using MPH quartic. Computer Aided Geometric Design, Vol. 20, pp. 469-492, 2003.

[17] J. Kosinka and B. Jüttler: $G^{1}$ Hermite Interpolation by Minkowski Pythagorean hodograph cubics. Computer Aided Geometric Design, Vol. 23, pp. 401-418, Elsevier, 2006.

[18] J. Kosinka and B. Jüttler: MOS Surfaces: Medial surface transforms with rational domain boundaries. Lecture Notes in Computer Science, Vol. 4647, pp. 245-262. Springer, 2007.

[19] J. Kosinka and B. Jüttler: $C^{1}$ Hermite interpolation by Pythagorean hodograph quintics in Minkowski space. Advances in Computational Mathematics, Vol. 30, pp. 123-140, 2009.

[20] R. Krasauskas and C. Mäurer: Studying cyclides with
Laguerre geometry. Computer Aided Geometric Design, Vol. 17, pp. 101-126, Elsevier, 2000.

[21] R. Krasauskas: Minimal rational parametrizations of canal surfaces. Computing, Vol. 79, pp. 281-290, Springer, 2007.

[22] R. Krasauskas: Branching blend of natural quadrics based on surfaces with rational offsets. Computer Aided Geometric Design, Vol. 25, pp. 332-341, Elsevier, 2008.

[23] M. Lávička and B. Bastl: PN surfaces and their convolutions with rational surfaces. Computer Aided Geometric Design, Vol. 25, pp. 763-774, Elsevier, 2008.

[24] D.T. Lee: Medial axis transformation of a planar shape. IEEE Trans Pattern Analysis and Machine Intelligence, Vol. PAMI-4, pp. 362-369, 1982.

[25] W. Lü and H. Pottmann: Rational parameterization of quadrics and their offsets. Computing, Vol. 57, pp. 135-147. Springer, 1996.

[26] H.P. Moon: Minkowski Pythagorean hodographs. Computer Aided Geometric Design, Vol. 16, pp. 739-753, Elsevier, 1999.

[27] T. Nishita, T. W. Sederberg, and M. Kakimoto. Ray Tracing Trimmed Rational Surface Patches. ACM SIGGRAPH Computer Graphics, Vol. 24, pp. 337-345, 1990.

[28] G. Nürnberger and F. Zeilfelder: Developments in bivariate spline interpolation. Journal of Computational and Applied Mathematics, Vol. 121, pp. 125-152, 2000

[29] M. Peternell, B. Odehnal, and M.L. Sampoli: On quadratic two-parameter families of spheres and their envelopes. Computer Aided Geometric Design, Vol. 25, pp. 342-355, Elsevier, 2008.

[30] M. Peternell and B. Odehnal: On Generalized $L N$-Surfaces in 4-Space. In: Proceedings of 'ISSAC08', pp. 223-230, 2008.

[31] M. Peternell and H. Pottmann: A Laguerre Geometric Approach to Rational Offsets. Computer Aided Geometric Design, Vol. 15, pp. 223-249, Elsevier, 1998.

[32] J. Peters, U. Reif: The 42 equivalence classes of quadratic surfaces in affine $n$-space. Computer Aided Geometric Design, Vol. 15, pp. 459-73, Elsevier, 1998.

[33] H. Pottmann: Rational curves and surfaces with rational offsets. Computer Aided Geometric Design, Vol. 12, pp. 175-192, Elsevier, 1995.

[34] H. Pottmann and M. Peternell: Applications of Laguerre geometry in CAGD. Computer Aided Geometric Design, Vol. 15, pp. 165-186, Elsevier, 1998.

[35] M. Ramanathan and B. Gurumoorthy: Constructing medial axis transform of planar domains with curved boundaries. Computer-Aided Design, Vol. 35, pp. 619-632, Elsevier, 2003.

[36] R.-H. Wang: Multivariate spline functions and their applications. Springer, 2001. 